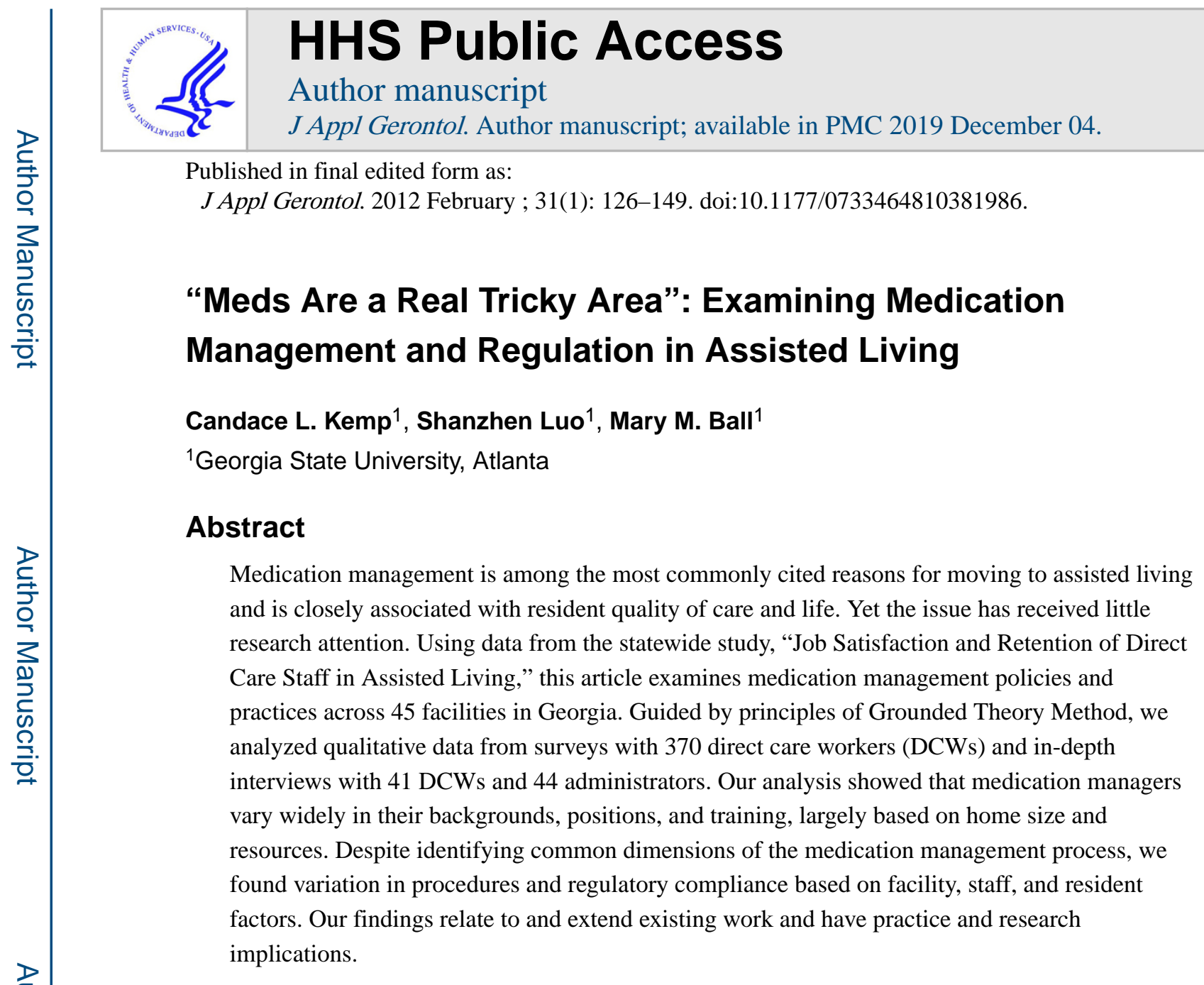

\title{
Keywords
}

assisted living; medication management; medication regulation; policy

Assisted living (AL) is the fastest growing long-term care (LTC) option in the United States (Golant, 2008; Mollica, Johnson-Lamarche, \& O’Keefe, 2005). Typically, older adults relocate to these nonmedical, residential care settings when they are unable to live independently and require help with activities of daily living (ADLs) and instrumental activities of daily living, including medication management (Ball et al., 2005; Ball, Perkins, Hollingsworth, Whittington, \& King, 2009). The need for assistance with medications is among the most frequently cited reasons for relocating to AL (Wizwer \& Simonson, 2006). Of the nearly one million individuals living in AL nationwide, nearly $81 \%$ receive medication help, from reminding to pouring and administering (The National Center for Assisted Living, 2010).

\footnotetext{
Reprints and permission: sagepub.com/journalsPermissions.nav

Corresponding Author: Candace L. Kemp, The Gerontology Institute, Georgia State University, P.O. Box 3984, Atlanta, GA 30302-3984, ckemp@gsu.edu.

Declaration of Conflicting Interests

The authors declared no potential conflicts of interest with respect to the authorship and/or publication of this article.
} 
Medication management is closely associated with AL residents' quality of life and has serious implications for health outcomes (Spore \& Mor, 1997; Young et al., 2008). It is complicated by a variety of factors and thus is a challenging issue in AL settings (Carder, Zimmerman, \& Schumacher, 2009). Estimates of the prevalence of Alzheimer's disease or another type dementia range from almost half (Ball, Perkins, Hollingsworth, \& Kemp, 2010) to $67.7 \%$ (Leroi et al., 2006). In addition, many residents need medication help because of declining visual acuity, glare, and contrast factors in print material, and lack of knowledge about the medications (Mitty \& Flores, 2007). Furthermore, drug regimens can be complex (Wizwer \& Simonson, 2006); estimates of AL residents' daily numbers of medications range from 3.8 to 6.2 (Reinhard, Young, Kane, \& Quinn, 2006). Drug regimens also often change over time and can include psychotropic drugs (Gruber-Baldini, Boustani, Sloane, \& Zimmerman, 2004; Wizwer \& Simonson, 2006).

Unlike nursing homes, which are medical environments, most AL settings rely heavily on direct care workers (DCWs), who typically are not licensed nurses, to manage medications (Reinhard et al., 2006; Spellbring \& Ryan, 2003). AL regulations vary by state (Mollica et al., 2005). In the majority of states, regulations simply stipulate that those who assist residents with medications must be "functionally literate" (Mollica, Sims-Kastelein, \& O'Keeffe, 2007). General training requirements for DCWs are typically minimal and vary by state and facility (Ball, Hollingsworth, \& Lepore, 2010a; Hawes, Phillips, Rose, Holan, \& Sherman, 2003; Mollica et al., 2007).

Research addressing medication management in AL is limited. However, in a recent national survey of AL administrators, Mitty (2009) found the majority of facilities have policies governing storage, documentation, and accounting for controlled drugs. Yet it is unclear how administrators and staff interpret and practice these policies or how and why, beyond different regulatory contexts, they might vary.

Existing research also highlights problems. In a national survey, $61 \%$ of AL licensing officials in 46 states and the District of Columbia reported that medication problems occurred frequently or very often (Mollica et al., 2005). Medication administration errors such as residents' medications being given in the wrong dose or at the wrong time are reported in the literature (Mitty, 2009; Young et al., 2008). In 2007, the Georgia Long-Term Care Ombudsman Program (2008) received 91 complaints related to medication management made by or on behalf of AL residents. These reports represent $8 \%$ of all complaints.

A 2-year survey examining medication problems in AL in four states (U.S. General Accounting Office, 1999) concluded that lack of training among unlicensed staff members, low pay rates, and high turnover rates were contributing factors. This finding suggests that industry and facility factors can influence significantly medication management practices. Existing studies report a variety of strategies developed by states to reduce medication problems in AL. These include offering additional training or enacting changes to nurse delegation (extending nurses' capacity; Reinhard, Young, \& Kane, 2003); developing a new comprehensive model for medication management and wellness care in collaboration with administrators, DCWs, residents, and family members (Meade, 2001); using a standardized 
system for packaging, storage, and documentation (Fitzgerald, 2004); and enhanced health information technology (Spiro, 2005).

The literature addressing medication management policy and practice in AL is limited (Clark, 2001, 2003; Mitty \& Flores, 2007; Reinhard et al., 2006). This exploratory research intends to expand current knowledge in this area by examining medication management in 45 AL settings in Georgia. Specifically, our aims are (a) to gain understanding of medication management in AL, including who manages medications, how facilities train staff who assist with medication, and the policies and practices associated with medication management, and (b) to identify factors that influence medication management in AL.

\section{Design and Method}

\section{Setting}

This study was set in Georgia, where AL residences are designated "personal care homes" and include a wide range of facilities. Georgia has 2,230 licensed AL homes with 30,169 beds (Georgia Long-Term Care Ombudsman Program, 2010). Although the majority of residents live in larger AL settings, the majority of homes are small (2-24 beds). Like 18 other states, Georgia does not have an assisted-living licensing category (Mollica et al., 2005). Available data suggest that Georgia residents resemble the national profile of an increasingly impaired and majority White and female population (Ball et al., 2000, 2005; Hawes, Rose, \& Phillips, 1999).

Georgia's AL regulations require that all persons who provide direct care to residents be at least 18 years of age and must complete first aid and CPR training within the first 60 days of employment. Regulations also specify that care staff complete at least 16 hours of continuing education annually in courses approved by the state Department of Human Resources (DHR, 2008). The content of this training varies widely across facilities (see Ball et al., 2010a).

Georgia regulations cover specific medication procedures, including ordering, storing, supervision of residents' self-administration, and documentation (see Georgia Department of Community Health, 2010). They do not address training for those who assist with medication. Like most states, Georgia regulations prohibit medication administration or provision of skilled services by facility staff (Hawes \& Phillips, 2000; Mollica et al., 2005). Thus state regulations allow DCWs to assist residents with self-administration rather than directly administering medication (see Mitty, 2009). Compared to some states (see Reinhard et al., 2003), at the time of the study Georgia had fewer guidelines and more limitations on assistance in self-administration and nurse delegation. ${ }^{1}$ Although regulations govern three administration methods (oral, topical, and injectable), this analysis focuses on oral administration. Regulations are specified below where relevant.

\footnotetext{
1. Legislation passed in the 2009-2010 session of the Georgia legislature, though, has expanded the GA Registered Professional Nurse Practice Act to allow unlicensed assistive nursing personnel to administer medications in AL settings (2010, Code Section 43-26-12 of the Official Code of Georgia Annotated).
} 


\section{Sample Selection and Data Collection}

To address our present research aims we drew on qualitative data derived from a federally funded statewide study, "Job Satisfaction and Retention of DCWs in Assisted Living," which involved a random sample of $45 \mathrm{AL}$ residences in Georgia stratified by size (18 small: 16-25 beds; 13 medium: $26-50$ beds; and 14 large: $51+$ beds). Using mixed methods, the broader study examined the meaning of job satisfaction for DCWs and factors influencing their satisfaction and retention (see Ball et al., 2010 for more details). The analysis reported here utilizes four data sources. The first source involved in-depth interviews with 44 facility administrators (one managed three facilities, two jointly managed two facilities, and in one, two were interviewed). Administrator interviews addressed all aspects of facility life, including descriptions of staff positions, responsibilities, training, and practices. Next, 370 DCWs, randomly sampled (stratified by employment status and shift from the 45 study homes), completed a survey, which collected information about personal characteristics and work experiences and included the questions, "Do you usually help with medications?" and if so, "What do you do?" Third, we used qualitative interview data from 41 DCWs (representing 35 homes) who were purposively selected according to age, gender, race, education, experience, shift, work status, and nativity. Information about medication management comes from responses to "Tell me about a typical work day, beginning with when you get to work." Finally, we drew on field note data from participant observation and informal interviewing in all homes. During observations researchers took particular note of medication management, clarifying observations through informal interviews. The project was approved by Georgia State University's Institutional Review Board. For purposes of anonymity, we use pseudonyms.

\section{Sample Characteristics}

The majority of residences were corporately owned (69\%) and for-profit (84\%). Approximately one in four was located in a rural area, including nearly half of the small homes. All large homes were in urban areas. Mean minimum and maximum monthly fees ranged from US $\$ 1763$ to US $\$ 2859$. Mean hourly wages ranged from US\$7.10 to US $\$ 9.83$. All large homes, $23 \%$ of medium-sized, and 39\% of small had an LPN or RN on staff.

The majority of residents were female (76\%). Most were White (95\%) and 65 years or above (94\%). Based on provider reports, $47 \%$ of residents had some degree of cognitive impairment, and $42 \%$ needed assistance with three or more ADLs. Most administrators were female, White, and had at least some college education. Administrators were employed an average of 13 years in LTC and 6.3 years in their current facility. Virtually all DCWs were female. More than half (57\%) were Black and most (82\%) were native born. They ranged in age from 18 to 75 years, with a mean of 40 . Education varied; 55\% had CNA training. DCWs were employed an average of 7.8 years in LTC and 2.5 years at their current facility.

\section{Data Analysis}

Our qualitative data analysis followed principles of grounded theory (Strauss \& Corbin, 1998). All authors participated in coding. Initially we used line by line, or "open coding," to look for emergent concepts based on the data and guided by our research questions. Open 
coding categories included, for instance, medication assistance procedures, documentation, ordering, storage, and training. As concepts were identified and compared, we categorized them thematically. Next, we conducted axial coding, which involved linking categories and making connections between the data that indicate relationships, conditions, context, and consequences (Strauss \& Corbin, 1998). We made charts and created memos for further analysis and to aid in interpretation. During axial coding, we noted, for example, how certain procedures varied by facility size or ownership as well as by resident frailty and compliance and staff competence. Based on this analysis, we offer a conceptual model representing the factors that influence the medication management process in AL. The qualitative analytic program Ethnograph 6.0 was used to store and manage the data. Descriptive statistics are used to illuminate the context of the qualitative data.

\section{Findings}

\section{Medication Managers' Job Preparation and Positions}

Overall, 65\% of DCWs' (261/400) reported that they routinely helped residents with their medications. Although the specifics of workers' involvement in medication assistance may differ, we refer to them collectively as medication managers. The proportion of DCWs involved in medication management varied by facility size, as did the specific task configuration of the role and often how DCWs were prepared for the job. In this section, we examine DCWs' preparation for medication management, the way that facilities allocated the various positions and tasks associated with the role, and the influences on both processes.

Preparation.-Table 1 presents information about the education and training, LTC experience, and facility tenure of the 261 medication managers by facility size. The proportion of medication managers without a high school education was higher in small homes $(28.4 \%)$ than in medium (16\%) and large $(9.5 \%)$. Those in small homes also were less likely to have care-related training: $43.2 \%$ were CNAs, compared to $52 \%$ in medium and $70.5 \%$ in large; none were LPNs, compared to $6.7 \%$ in medium and $5.7 \%$ in large; and none had medication certification, compared to $2.7 \%$ in medium and $9.5 \%$ in large. A minority of medication managers were LPNs (10.5\%) or had medication certificates (11.4\%). As seen below, whether medication managers had medication certification, a credential typically offered by large AL corporations, was related to the size and ownership of the facilities where they were or had been employed.

Only $9.6 \%$ of medication managers had worked in LTC for 1 year or less; $52.7 \%$ had been in the field 5 or more years. Almost one third (29.9\%) of managers had worked in their facilities 1 year or less; the proportion was higher in small homes (42\%), compared to medium (20\%) and large (27.6\%). In general, a facility's hiring practices, also tied to size, drives the education, experience, and skill level of its DCWs, including medication managers (see Ball et al., 2010a.)

Irrespective of DCWs' prior experience and training, all facilities provided some training related to medication assistance. In all facilities, medication training involved job "shadowing," where new hires, or other DCWs new to medication management, learned on 
the job from more senior staff. In $75.5 \%$ of facilities-including all but one of the small, all of the medium, and four of the large_-job shadowing was the only training.

Some variation was evident in the type of worker providing the on-the-job training. For example, in at least 5 of the 18 small homes, the person being shadowed was the owner or other supervisory staff. In one medium-sized, corporately owned facility where shadowing was the sole type of training, trainees were required to pass a test before beginning medication management.

In seven corporately owned facilities (one small and six large), additional classroom instruction was mandated and provided at the corporate level. One large corporation, for example, required all medication managers to participate in a 3-day training course and pass a test. Corporate training typically was provided off-site and was comprehensive. Three large facilities supplemented job shadowing with in-house classroom instruction.

Administrators in general emphasized the importance of medication training for a variety of reasons, including the critical nature of the job, the skill level of new hires, and the regulatory process. The director of a large facility dedicated to dementia care explained,

Meds are a real tricky area in AL but we have a real good training program and we are about to up that in a little bit. You know, most people that come into this field are not skilled, they haven't been to college ... They come into this field with little or no education. And we make sure that these girls that do that are very well trained because you definitely don't want any medication errors.

The director of a medium home, who hoped to begin classroom training, expressed the ambivalence of some administrators regarding the value of rigorous training:

We are going to be starting, they have to go to an 8-hr class and at the end of the eight hours they take a test, just on administering medications. These guys are not RNs and neither am I so I feel like we shouldn't be able to give them, but then again, anyone can read a bottle and do what it says.

The owner of a medium home in a rural area voiced the low bar of functional literacy for medication managers, "I have to have you where you can read and write because sometimes you have to administer medicine."

A minority of DCWs reported they lacked adequate training in medication management. For the full sample, $19 \%$ of workers indicated a need for additional training in this area. Among medication managers, $12 \%$ named medications as a training need. One said, "There's always so much to learn about different meds."

Positions and task configuration.-All DCWs in these homes were universal workers (see also, Ball, Hollingsworth, \& Lepore, 2010b), meaning they performed a variety of tasks. A facility's organizational structure, which largely depends on size, determined the number and type of DCW positions and the configuration of tasks workers performed. In general, the smaller the facility, the fewer and more universal were the positions. It follows then that although $65 \%$ of all DCWs were medication managers, the proportion of workers 
performing this role was higher in small facilities (94\%) compared to medium (68\%) and large $(51 \%)$.

Medication managers held three types of positions: resident assistant (RA), lead RA or shift supervisor, and medication technician or med tech. The large majority (72\%) of small homes had only RAs, and in 55\%, all RAs were medication managers. As one director said, "Just whoever is on the shift. That is the one who gives out the medications." In 70\% of medium homes, only select RAs or shift supervisors were medication managers. Selection of DCWs for this position depended on personal traits or additional training. One director explained her rationale,

We also recognize that there are some caregivers who can't do meds. We have several, they are great caregivers but they are not anal enough to be able to do meds. To do meds you have to be precise and exact and there is no room for error.

The director of another medium home said, "We limit the number of people who give medication and we train them and have it certified in their folder before they can give it." The choice of medication managers was most selective in the 14 large homes: 6 used only certified med techs; 4 , only shift supervisors; and 2, only skilled nurses.

Other data suggest that selection of workers for medication management related to the controlled nature of the medications. The owner of a large home explained, "The shift supervisors are the only ones that give out medicine because we have a lot of narcotics in our building."

Data further indicate that DCWs' attitudes toward mediation management had some influence over who held this position. Several workers expressed a reluctance to take on this role, either because of potential stress or diversion from a more preferred task. One worker said,

I don't want to pass meds at this job or any other job unless I am an RN because I can be held accountable if somebody doesn't get their medicine and some residents don't want to take their medicine.

The position that medication managers held influenced the extent to which their job involved medications relative to other tasks. The principal job of med techs, for example, was medication management although they performed other tasks, as the director of a large facility explained:

This group of staff members are charged with making sure the residents are receiving the right medication with the right dose at the right time. That is their primary job ... However, as part of the team that works with the care managers, they do pitch in.

Although med techs reported an average of 7 daily tasks, medication assistance comprised the bulk of their day. In contrast, RAs, with an average of 8 tasks, spent considerably less time in medication management compared with other chores.

The shift a medication manager worked also influenced task configuration, with mornings typically involving greater involvement in medications. A DCW in a medium home 
commented: "Lord, when I first come in I start dispensing medication. That is the biggest part of the job, especially in the morning because that is when we give out the most medication."

Data indicate that in a minority of facilities DCWs not designated as medication managers assisted residents on a regular basis with PRN (Latin for "pro re nata" meaning "when necessary") medications. The director of a medium home reported about two night shift workers: "They don't really give medicine at night. The only thing they give, they will give some Tylenol if they [residents] have a headache or if they can't sleep they might want a sleeping pill."

\section{The Medication Management Process}

Across study homes, managing medication was a process involving three main dimensions: (a) maintaining appropriate inventory of medication, (b) storing, and (c) assisting residents. Figure 1 illustrates the process and its dimensions. Dimensions were connected such that policies and procedures of each affected and informed one another and the entire process. Precise record keeping and effective communication were integral components of the overall process and keys to proper medication management (i.e., follows specified procedures).

Figure 2 illustrates the factors that influence the medication management process in AL. As can be seen, state AL regulations influence facility policies and procedures and DCW hiring and training practices, which are simultaneously influenced by a facility's size, organizational structure, resources, location, and ownership. In turn, facility factors influence who manages medications - their training, position, and the nature of their involvement in the process-all of which connect and influence their work habits, strategies, and procedural compliance. Factors relating to facilities and medication managers join with resident factors (functional status, compliance, and drug regimens) to influence how medications are managed in AL, including levels of safety and compliance. Below we explicate the medication management process, its dimensions, facility approaches to management, and relevant state regulations and other influential factors.

Maintaining appropriate inventory.-State regulations require that a facility's admission agreement specify the person responsible for initial acquisition and refilling of prescribed medications, and all homes complied with this rule. Exactly who and how many individuals had this responsibility depended largely on facility organizational structure and size. In small homes, it was common for administrators and RAs to oversee ordering and communicate with doctors and pharmacies. In homes with more complex organizational structures, supervisors or med techs were typically, but not exclusively, responsible.

Ordering involved knowing residents' medication needs, which included being aware of prescription changes, and knowing how much and what was on hand. A med tech from a large home spoke of her duties saying it was necessary to "see if you need to reorder medication from pharmacy" and communicate with the "doctor to reorder." In one small home the administrator explained, "The supervisor, of course, is in charge of ordering medications ... she also checks the medication sheets each and every day that she comes in to make sure that, you know, everything is right" 
"Medication sheets" are essential to ordering. Such documentation is required by the state and a critical component of annual surveys. Regulations specify that homes maintain records of all medications, including the observed drug and dosage, date and time given, and the name of the staff person responsible for supervising or assisting. All homes typically recorded this information in a book identified as the "Medication Administration Record" or MAR. Those who ordered medications relied on the MAR to track medications, including what is prescribed, delivered, and given to residents. One RA explained, "I go through the MAR to make sure that what the doctor prescribes, I have it."

Accurate documentation throughout the medication management process was essential to maintaining appropriate inventory. Accuracy began with effective training and awareness of regulations. One administrator noted, "They have to have training on documentation [and] medication guidelines. The state requires us to meet these standards."

State regulations direct that homes cannot require residents to obtain medications from a specific pharmacy or pharmacist. Available data suggest that most homes ordered from a single pharmacy, with convenience as a possible factor. In at least a few homes pharmacy selection reflected family member or resident choice.

Storing.-Storage refers both to type of packaging and where medications are stored. Regulations do not stipulate type of packaging, but all large and most medium and small homes reported using "medication on time" systems where medications come prepackaged in bubble packs. Two medium homes used bottles exclusively and the remaining homes used a combination.

Regulations require that medications be kept under lock and key at all times in whatever location they are stored. All homes reported complying with this regulation. Only one, a medium home, stored medications in residents' rooms. A medication manager explained, "Each resident has their own medication in a locked drawer in their own room." All large homes, five medium-sized (38\%), and five small homes (28\%) had a designated medication or wellness room and/or a medication cart. In the remaining homes medications were stored in various locations: within the main office, the kitchen, activity or TV rooms, and, in one instance, the beauty shop.

Despite having a locked "med closet," field note data from a medium-sized, not-for-profit home noted, "meds were just on a shelf in the dining room and were not locked away." In the same home, an RA expressed concern over a coworker who, "loses medications all the time and does not lock the meds away appropriately." She identified lack of "protocol" around medications as a factor contributing to violations.

Assisting residents.-State regulation stipulates that when residents are incapable of self-administering, staff may assist by reminding, reading medication regimens, checking dosages, and assisting in getting fluids or pouring, opening, or punching-out the medication container. Assisting with medications in the study homes involved five steps: (a) getting meds ready, (b) giving to residents, (c) explaining meds, (d) ensuring compliance, and (e) documenting. 
Getting ready.-Getting medications ready typically began by "checking the MAR." Preparation often involved "pulling meds", particularly when med carts were used. In some large and medium-sized homes medications were "pulled" in advance. In a large home, a med tech explained, "I come in the morning. Medicines are pulled the day before."

The majority of homes kept medications in original containers during the pulling process. An RA in a large corporately owned home explained, "We are not supposed to open medicine when the resident is not there." Her words reflect the state regulation that medications be kept in original containers with original label intact. However, in a few medium homes, medication managers reported or were observed "popping" medications into cups in locations away from residents prior to assisting.

Getting ready sometimes involved "crushing meds." One caregiver explained, "We crush medicine for people who cannot swallow." Residents' cognitive or physical ability largely determined whether medications were crushed.

Giving medications.-Once ready, the next step involved giving medication to residents. This dimension includes how medications were passed as well as when and where. Although the majority of homes used bubble packs, we identified five different approaches to giving medications: (a) handing the package to residents, (b) popping medications into residents' hands, (c) popping medications into a cup or other receptacle before giving to residents, (d) putting medications in residents' mouths, and (e) giving medications with a spoon.

The main factor influencing the approach was residents' physical and cognitive abilities. One RA explained, "We hand the bubbles to the residents and if they don't have a way to open the bubble, we just put it in their hand. We have to follow state protocol on all of those." This facility's procedure was influenced by efforts to conform to regulatory compliance, which could be upheld because of their residents' ability to self-administer.

In some homes, assisting meant also administering because of resident need. The administrator from a small home described a range of need,

If you are unable to take the medicine out [of the package], I will assist you in getting it into your hand. If you are unable to read your meds or if you are dysfunctional to where you just can't do it, that is when I will do it for you.

"Do[ing] it for" residents was noted in homes with frailer residents. One care supervisor explained, "Arthritis for instance, we administer. We put meds in a little cup, take into their room, hand it to the resident or put it in the resident's mouth for those who had strokes and have limited motion." An RA commented, "Some [dementia] residents get confused and staff have to put meds in mouth." "Crushed" medications were mixed in applesauce, jam, or yogurt, and served with a spoon.

Facility policies and practices also influenced the approach. Eighteen homes ( 8 small, 6 medium, 4 large) reported using cups or similar receptacles. An RA explained, "I put pills in a cup. I don't know if that is right, but I do what they tell me to do." Reports of putting medications directly in residents' mouths were common, but in at least one home, staff were "not allowed to put in [a resident's] mouths" in case "they vomit." 
In at least two homes (small and medium), the administrator's philosophy on resident independence was influential. Staff reported they "prefer to let residents do" if possible. An administrator elaborated, "The thing is, you are trying to get them to develop independence skills, independently taking the right meds and the right doses."

Medication assistance frequently coincided with mealtimes, which influenced where meds were passed. A care supervisor from a medium home described the typical approach, "In the day, they are given in the dining room because they are given with breakfast, lunch and dinner. But at night, they're given in the rooms." In seven homes all medications were passed in residents' rooms. Reasons for this approach included storage location, a perception of mealtimes as "too confusing," and, a policy of " total medication privacy," to avoid violating "the privacy and dignity of residents." Influenced by residents' functional status, 11 homes primarily passed medications in common areas or required residents to come to central locations. Likely owing to size, in three small and two medium-sized homes, including one where resident preference was influential, medication managers reported they "go to [the] resident wherever they are."

Explaining.-Although not universally practiced, explaining was a step noted in three small and one large home. Medication managers reported explaining medications to residents either before or while being given. In the small homes, they tell residents "what it is for ... only when [a] resident asks." In the large home, staff "let [residents] know what they are getting" as part of their regular routine.

Ensuring compliance.-Medication managers reported that while some residents refuse medication others "play tricks" and "act like they took it" but "throw it away." In these instances, medication staff reported the "need to stand and watch to be sure they don't throw it away." Some examined residents' mouths. As will be seen, ensuring resident compliance was a key challenge, influenced by resident factors. Responses to noncompliance were influenced by staff and facility factors.

Documenting.-Most medication managers reported documentation as a regular procedure. One explained, "[I] mark off the medicine I am going to dispense and initial in the record book when I have given the medicine." Refusal also was documented. As one RA noted, "If they refuse, I initial." Two homes reported problems with not "signing off." In both cases, individual staff members failed to follow procedure.

\section{Key Challenges}

Administrators and medication managers encountered some key challenges regarding medication management. Challenges revolved around safety, handling narcotics, and resident compliance and were influenced by facility, staff, and resident factors.

Safety.-The main safety concern voiced by administrators and medication managers was ensuring "residents get the medication at the right time, the right medicine, and the right dosage." Residents' complex and changing drug regimens could interfere with this goal. Changes were problematic if undocumented or communicated ineffectively. Failure to check or follow documentation was reported in one small and three medium-sized homes. The 
small home administrator offered this account of a resident whose Haldol dosage was halved,

They wrote it in the med book and the communication book [but] the girl on third shift gave her a whole $5 \mathrm{mgs}$. Did it hurt her? No. It is not what is called a medication error, but the doctor's orders were changed and it was in the book ... She didn't [follow] the procedure.

This mistake was not life threatening, but the potential existed. For example, field notes from the home where medications were improperly stored reported that a resident who had a stroke,

had not been receiving the medication her doctor prescribed, Plavix. The med had been delivered to the facility ... and never dispensed to the resident. It was listed on her MAR sheet ... but ... sat on the shelf for a month before it was discovered.

Multiple workers overlooked the change, which hints at systemic communication problems.

Most homes had strategies for promoting medication safety. As discussed earlier, in homes with more complex organizational structures only certain workers managed medications. All homes used MARs, but some medium and large homes enhanced communication using logs where staff "write down any pertinent information for their shift." Some staff described additional communication. A med tech explained, "I talk with the med tech that is going off and I do a review of what has happened."

Almost all administrators attempted to simplify medication management. Use of bubble packaging was one way. Yet, as one administrator commented, "they still have to know who they are talking to and make sure they have the right person's medication bubble pack. It should be idiot proof but it is not." One key to good medication management is knowing residents, which is easier in smaller homes. Strategies to assist staff included putting residents' pictures on medication sheets, organizing medications alphabetically, and using color-coded packaging to indicate administration times.

In the majority of homes medication managers described the importance of "checking" for "right person" and the "right medication. One said, "If you give the wrong medication it can be devastating ... You check and you double-check and you triple-check." Checking sometimes meant asking for guidance from the administrator or RN.

Handling narcotics.-Handling narcotics was an ongoing challenge. Instances of theft were reported in four small and three medium-sized homes, but theft was possibly more widespread. One administrator said of theft in her small home, "it's medicine on time and if it's gone, it's gone and it's very obvious to the next person coming in." In other instances, medication managers signed off on medications that were not given. In a medium facility with a history of theft, the administrator described this method "If she writes it in the book, 'I gave it to Sam or I gave it to John', she knows they have Alzheimer's so [I] cannot question them." 
Factors influencing drug theft among care staff included having access to medication and an opportunity to work independently, which were more likely to happen in smaller facilities and on night shift, when oversight was limited. Drug addiction factored in also and was a problem in locations with limited hiring pools.

Most attempts to prevent theft began with hiring. One administrator explained, "I have to be really careful screening them." She watches for those who "are too anxious to get with the drugs." Criminal background checks are part of the hiring process, but most homes added mandatory drug testing.

Beyond hiring, in most homes, medication management included counting narcotics. One administrator elucidated, "On each shift they have to do the narcotic count ... if they are ever off, they have to call me and they can't leave." In some homes only certain individuals had access to medications.

Resident compliance.-Compliance varied by resident's cognitive impairment and mental health. An RA in an all dementia facility noted, "Up in here [DCU] it is so hard trying to get them to take those meds. Some days they might cooperate but the majority of the time you try to get them in them."

Being familiar with residents helped some medication managers overcome noncompliance. A med tech explained, "I know what to say or how to approach a resident to get them to take their medication 98 percent of the time." Time, patience, and encouragement or "coaxing" were further strategies. A field note excerpt illustrates, "Pam gave Irving his meds but he refused to swallow. He even spat a capsule ... She coaxed and encouraged him. It took a good 20 minutes." A common practice was to "document and try again later."

Noncompliance was viewed as a resident rights issue in most homes. One administrator explained, "Whether it's daily cleaning or whether it's taking their medications, we can't make them do anything." However, in a small home with high rates of mental illness, according to the administrator, compliance was not optional: "I can't make you take it, but if you refuse to take your meds, you have twice to do it for me. I give you two times and I shoot you out of here."

\section{Discussion}

Our research examined medication management in $45 \mathrm{AL}$ residences across Georgia in an effort to understand who manages medications, how staff are trained, and the policies and practices associated with the medication management process as well as to identify influential factors. Our analysis shows that medication managers vary widely in their backgrounds, positions, and training, largely based on a home's size and resources. Despite procedural variation, we identified three main dimensions of the medication management process, which were common across all homes. Variation in this process was influenced by factors relating to state regulations, facilities, staff, and residents. Administrators and medication managers identified safety, handling narcotics, and resident compliance as key challenges. Overall our findings relate and add to existing work and have practice and research implications. 
To begin, our findings on medication managers suggest that those in smaller homes typically had less formal education, care experience, and training than those in larger homes. Facility resources were also important in this regard as only corporately owned homes reported significant classroom training. Training has been identified as a strategy for reducing medication management problems (Mitty, 2009; Spellbring \& Ryan, 2003). Our findings suggest that medication managers need to pay attention to detail, communicate effectively, and be aware of and follow procedure to do their jobs effectively. Training in these areas is fundamental to developing good medication managers.

In smaller homes almost all DCWs were medication managers. Although these workers had fewer qualifications, they were typically responsible for a greater number of work tasks in addition to medication management. However, they cared for fewer residents, which likely increased familiarity. Knowing residents was important for safety and compliance reasons and may be easier to achieve in the smaller settings.

Medication management can be time consuming in AL (Young et al., 2008), particularly in larger homes. Medication managers in these settings typically had fewer job tasks, but more residents to assist, which could mean added repetition and job stress as well as less familiarity with residents. However, limiting who gives medications, as many larger homes did, was a strategy to promote procedural compliance and safety in medication management.

Our data indicate that in certain homes PRNs are treated differently than other categories of medication. These medications are given by those who are not ordinarily considered medication managers, a practice which could be a safety issue because of inadequate training. This is an area requiring further study.

Our examination of the medication process revealed variation within the three dimensions. Facility size and resources influenced variations in medication storage. Ordering procedures varied in terms of who (i.e., position) was involved. Our limited data suggest a few homes ordered from multiple pharmacies because of resident or family member choice. Although this practice may be a consumer choice issue, Crutchfield (2000) suggests that the use of multiple pharmacies by a given AL facility makes the management process more difficult for medication managers. Future research should examine this issue.

Assisting residents with medication management had the most variation across study homes, particularly in terms of giving medications. Variation was based on facility, medication manager, and resident factors, with the latter being the most influential. Georgia regulations indicate that AL staff can assist residents with self-administration rather than administering medications. Yet the distinction can be unclear. As Mitty (2009, p. 107) explains, "the difference between 'assist with' and 'administration of' medications is often based on what a regulation says ... by observation, however, the 2 acts can look the same." Our findings suggest that in the majority of homes, residents' functional abilities influenced whether medication managers were assisting or administering. Crushing medication or putting it in residents' mouths can be considered the latter and was common. The implications of assisting versus administering and interpretation of regulations require further consideration. 
Carder (2008) describes medication management in AL as a "social situation" involving different actors (i.e., licensed and unlicensed professionals and residents). She identified safety and autonomy as two discourses that dominate existing policy, practice, and research contexts. Our findings speak to both. Participants identified safety and resident compliance as challenges. Although refusing to take medications typically was viewed as a resident right, doing so was more complicated for residents with mental illness or cognitive impairment. Other research has addressed the conflict between resident safety and autonomy in AL (Ball et al., 2005).

Carder and colleagues (2009) suggest that a resident's ability and background influence their different needs and preferences regarding medication management. Our findings on the influence of residents' functional status and preferences resonate with their work. In addition, we found administrator and staff philosophy influenced medication management and was related to how, if at all, facilities promote resident independence and respond to noncompliance (see also Carder et al., 2009).

Participants reported routine documentation, but there were a few reports of failing to document appropriately. These practices were influenced by individual medication manager's work habits and procedural compliance, particularly inattention to detail. Improving work habits and compliance could be accomplished through training and accountability systems. In larger homes the practice of limiting who manages medication was a strategy to promote good medication management. In smaller homes or those with fewer resources, hiring pools limited available staff and this strategy may have limitations.

Findings point to challenges with handling narcotics. Although little discussion of these matters exists in previous research (but see Perkins, Ball, Whittington, \& Combs, 2004), this issue may increase in importance as the AL industry continues to grow and change. A study of behavioral symptoms in AL in four states found more than two thirds of residents had a mental health diagnosis and half were prescribed psychotropic drugs (Gruber-Baldini et al., 2004). Resident populations also are increasingly impaired (Golant, 2008), which may mean more frequent use of hospice services and greater likelihood of complex drug regimens, including the use of narcotics. Preventing drug theft remains a challenge and requires further investigation.

Findings raise issues about standardization. Mitty (2009, p. 111) suggests med tech certification should be "uniform and nationwide", which she argues would "assure a level of competence" and offer workers job security and mobility. Another possibility would be to standardize medication practices across the industry to maintain accountability and accuracy of medication management. However, standardization would require national regulation of $\mathrm{AL}$ and could move homes further away from social (as opposed to medical) models of care. Doing so could be difficult to enforce and too inflexible, especially considering the limited resources many AL residences have and the fact that individual residents have different functional abilities and preferences (Carder et al., 2009). If or how standardization might work, especially in smaller homes with fewer resources, are avenues for further research. 


\section{Strengths, Limitations, and Future Directions}

Although the present analysis has considerable value (e.g., data come from a statewide study; analysis draws on multiple data sources; and its focus on an important underexamined topic), limitations exist. The wider study was not specifically designed to research medication practices. Thus, in some cases, data are missing or incomplete. Resident and family member perspectives are absent and exact numbers of residents who need medication services and their drug regimens were not accessible. Another limitation is the exclusion of facilities with fewer than 16 beds-settings which may face unique challenges. A final limitation is that the study only considers Georgia.

Despite limitations, our exploratory research can be used as groundwork for further research. In addition to the suggestions identified above, future researchers might wish to take residents' and family members' perspectives into account. Further studies also should identify the significance of having an RN or LPN on staff and medication training in AL. As the population continues to age and the AL industry grows ever-more popular and diverse, additional research will be required to help promote quality medication management practices and enhance residents' quality of life and improve DCWs' work experiences.

\section{Acknowledgments}

The authors thank Molly M. Perkins, Patricia Clark, Carole Hollingsworth, and Frank J. Whittington for their support. They are also very grateful to all those who participated in the study.

Funding

The authors disclosed that they received research support. Data for this article come from the study, "Job Satisfaction and Retention of Direct Care Staff in Assisted Living " funded by the National Institute on Aging (1R01 AG021183; Mary M. Ball, principal investigator).

\section{Bios}

Candace L. Kemp, $\mathrm{PhD}$, is an assistant professor in the Gerontology Institute and the Department of Sociology at Georgia State University. Her research agenda includes recent work on aging, intimacy and family life, staff, resident and family members' experiences in assisted living, and planning for later life.

Shanzhen Luo, MA, is a research assistant in the Gerontology Institute at Georgia State University. Prior to joining the research team in 2009 , she earned her graduate degree from the Institute. Her primary research interests include health and aging, long-term care, aging policy development in East Asian countries, and postretirement planning.

Mary M. Ball, $\mathrm{PhD}$, is an associate research professor in the Gerontology Institute at Georgia State University. Her research for the past 14 years has focused on assisted living with a special interest in the quality of life of residents and care staff and the experiences of low-income and minority residents. 


\section{References}

Ball MM, Hollingsworth C, \& Lepore MJ (2010a). Hiring and training frontline workers In Ball MM, Perkins MM, Hollingsworth C, \& Kemp CL. Frontline workers in assisted living. Baltimore: Johns Hopkins University Press.

Ball MM, Hollingsworth C, \& Lepore MJ (2010b). "We do it all": Universal workers in assisted living In Ball MM, Perkins MM, Hollingsworth C, \& Kemp CL. Frontline workers in assisted living. Baltimore: Johns Hopkins University Press.

Ball MM, Perkins MM, Hollingsworth C, \& Kemp CL (2010). (Eds.), Frontline workers in assisted living. Baltimore, MD: Johns Hopkins University Press.

Ball MM, Perkins MM, Hollingsworth C, Whittington FJ, \& King SV (2009). Pathways to assisted living: The influence of race and class. Journal of Applied Gerontology, 28, 81-108.

Ball MM, Perkins MM, Whittington FJ, Hollingsworth C, King SV, \& Combs BL (2005). Communities of care: Assisted living for African American elders. Baltimore: Johns Hopkins University Press.

Ball MM, Whittington FJ, Perkins MM, Patterson V, Hollingsworth C, King SV, et al. (2000). Quality of life in assisted living facilities: Viewpoints of residents. Journal of Applied Gerontology, 19, 304-325.

Carder PC (2008). Managing medication management in assisted living: A situational analysis. Journal of Ethnographic and Qualitative Research, 3, 1-12.

Carder PC, Zimmerman S, \& Schumacher JG (2009). Understanding the intersection of individual needs and choices with organizational practices: The case of medication management in assisted living. The Gerontologist, 49, 463-473. [PubMed: 19491359]

Clark TR (2001). Medication use in assisted living: A review of published reports. Consultant Pharmacist, 16, 1036-1044.

Clark TR (2003). Medication use and pharmacist impact in assisted living facilities. Retrieved August 10, 2010, from http://www.ascp.com/public/pr/assisted/2003/rximpact.pdf

Crutchfield DB (2000). Managing medication and independence in assisted living. Nursing Home Long Term Care Management, 49(3), 46-48.

Fitzgerald SL (2004). ADL Focus: Managing meds. Assisted Living Today, 11(4), 23-25.

Georgia Department of Community Health. (2010). Rules and interpretive guidelines for personal care homes. Retrieved August 10, 2010, from http://dch.georgia.gov/vgn/images/portal/ cit_1210/24/41/156758976PCHRulesIGsFeb242010.pdf

Georgia Long-Term Care Ombudsman Program. (2008). 2007 Annual Report. Atlanta, GA: Office of the State Long-Term Care Ombudsman.

Georgia Long-Term Care Ombudsman Program. (2010). Unpublished raw data.

Golant S (2008). The future of assisted living residences: A response to uncertainty In Golant S \& Hyde J (Eds.), The assisted living residence: A vision for the future (pp. 3-46). Baltimore: Johns Hopkins University Press.

Gruber-Baldini AL, Boustani M, Sloane PD, \& Zimmerman S (2004). Behavioral symptoms in residential care/assisted living facilities: Prevalence, risk factors, and medication management. Journal of the American Geriatrics Society, 52, 1610-1717. [PubMed: 15450035]

Hawes C, \& Phillips CD (2000). High service or high privacy assisted living facilities, their residents and staff: Results from a national survey. Washington, DC: U.S. Department of Health and Human Services, Office of the Assistant Secretary for Planning and Evaluation, Office of Disability, Aging, and Long-Term Care Policy.

Hawes C, Phillips CD, Rose M, Holan S, \& Sherman M (2003). A national survey of assisted living facilities. The Gerontologist, 43, 875-882. [PubMed: 14704387]

Hawes C, Rose M, \& Phillips C (1999). A national study of assisted living for the frail elderly: Results of a national survey of facilities (Prepared by the U.S. Department of Health and Human Services). Washington, DC: U.S. Department of Health and Human Services. 
Leroi I, Samus Q, Rosenblatt A, Onyike C, Brandt J, Baker A, et al. (2006). A comparison of small and large assisted living facilities for the diagnosis and care of dementia: The Maryland Assisted Living Study. International Journal of Geriatric Psychiatry, 22, 224-232.

Meade V (2001). A new comprehensive model for assisted living medication management and wellness care. Consultant Pharmacist, 16, 638-652.

Mitty E (2009). Medication management in assisted living: A national survey of policies and practices. Journal of the American Medical Directors' Association, 10, 107-114. [PubMed: 19187878]

Mitty E \& Flores S (2007). Assisted living nursing practice: Medication management: Part 2, Supervision and monitoring of medication administration by unlicensed assistive personnel. Geriatric Nursing, 28, 153-160. [PubMed: 17561014]

Mollica R, Sims-Kastelein K, \& O'Keeffe J (2007). Residential care and assisted living compendium. Prepared for the U.S. Department of Health and Human Services. Washington, DC: National Academy of State Health Policy http://aspe.hhs.gov/daltcp/reports/2007/07alcom.htm

Mollica R, Johnson-Lamarche HS, \& O'Keefe J (2005). State residential care and assisted living policy: 2004. National Academy for State Health Policy: Portland, ME. Retrieved August 10, 2010, from http://aspe.hhs.gov/daltcp/Reports/04alcom.htm

Mollica R, Sims-Kastelein K, \& O’Keeffe J (2007). Residential care and assisted living compendium (Prepared for the U.S. Department of Health and Human Services). Washington, DC: National Academy of State Health Policy.

The National Center for Assisted Living. (2006). Assisted living resident profile. Retrieved from http:// www.ahcancal.org/ncal/resources/Pages/ResidentProfile.aspx

Perkins MM, Ball MM, Whittington F, \& Combs B (2004). Managing the care needs of low-income board-and-care home residents: A process of negotiating risks. Qualitative Health Research, 14, 487-495.

Reinhard SC, Young HM \& Kane RA (2003, June). Rutgers report on nurse delegation of medication administration for elders in assisted living. New Brunswick, NJ: Rutgers Center for State Health Policy. Retrieved August 10, 2010, from http://www.cshp.rutgers.edu/PDF/Nurse\%20Delegation $\%$ 20of\%20Med\%20Admin\%20for\%20Elders\%20in\%20AL.pdf

Reinhard SC, Young HM, Kane RA, \& Quinn W (2006). Nurse delegation of medication administration for older adults in assisted living. Nursing Outlook, 54(2), 74-80. [PubMed: 16597525]

Spellbring AM, \& Ryan JW (2003). Medication administration by unlicensed caregivers: A model program. Journal of Gerontological Nursing, 29(6), 48-54. [PubMed: 12830656]

Spiro R (2005, September/October). E-prescribing in assisted living: Keys to success. Assisted Living Consult, 26-28.

Spore DL, \& Mor V (1997). Inappropriate drug prescriptions for elderly residents of board and care facilities. American Journal of Public Health, 87, 404-409. [PubMed: 9096541]

The State of Georgia, Department of Human Resources. (2009). State regulation on personal care homes. Retrieved August 10, 2010, from http://rules.sos.state.ga.us/docs/111/8/62/21.pdf

Strauss A, \& Corbin J (1998). Basics of qualitative research: Techniques and procedures for developing grounded theory (2nd ed.). Thousand Oaks, CA: Sage.

U.S. General Accounting Office. (1999). Assisted living quality of care and consumer protection issues in four states. Washington, DC: GAO/HEHS-99-27.

Wizwer P, \& Simonson W (2006, November/December). Complex medication regimens call for help with medication management. Assisted Living Consult, 26-30.

Young H, Gray S, Mc Cormick W, Sikma S, Reinhard S, Trippet L, et al. (2008). Types, prevalence, and potential clinical significance of medication administration errors in assisted living. Journal of the American Geriatrics Society, 56, 1199-1205. [PubMed: 18482296] 


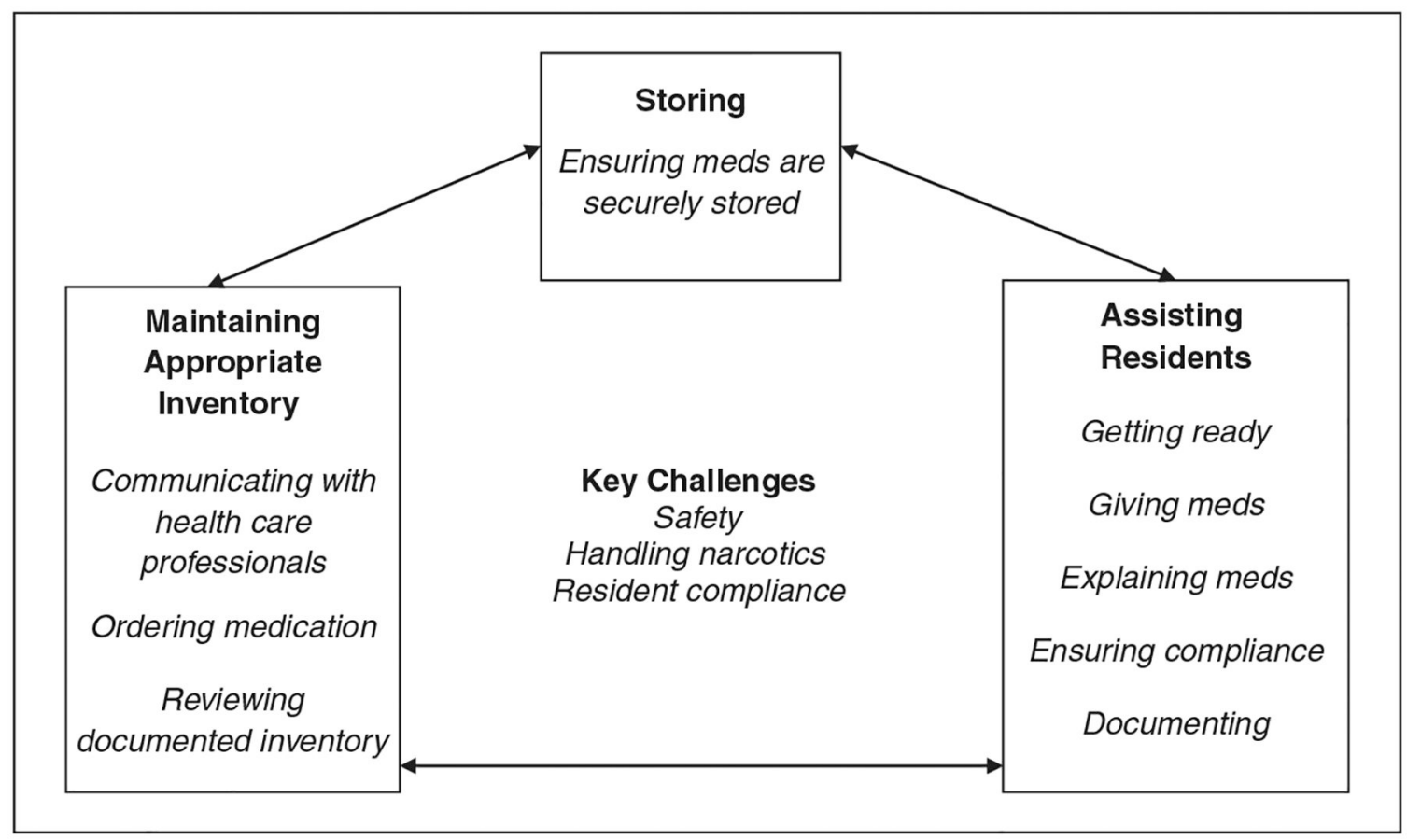

Figure 1.

Dimensions of the medication management process 


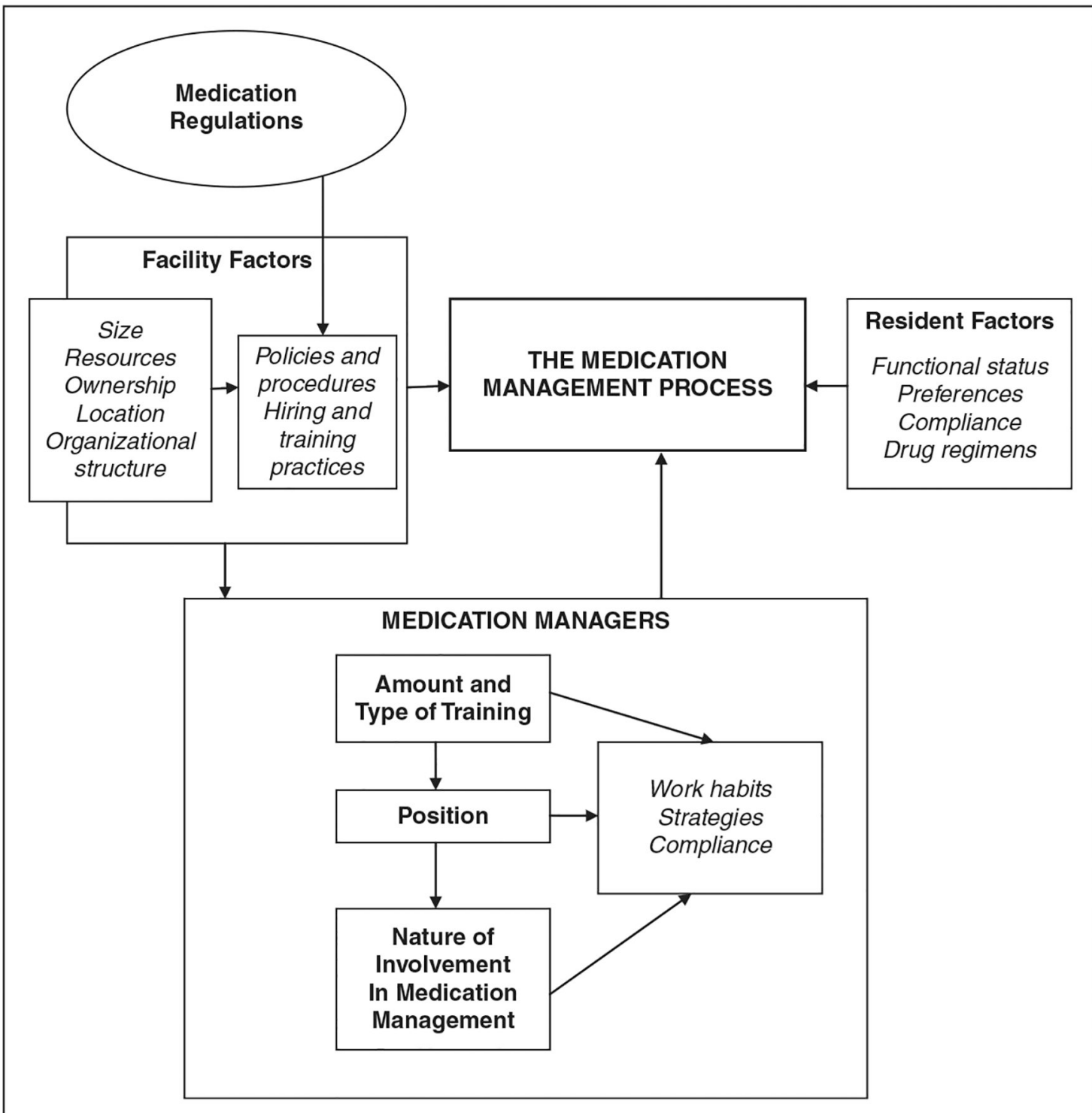

Figure 2.

Factors influencing the medication management process 
Table I.

Selected Demographic and Work Characteristics of DCWs Performing Assistance With Medications by Facility Size $(N=261)$

\begin{tabular}{|c|c|c|c|c|}
\hline Characteristic & $\begin{array}{c}\text { Small } \\
n=81(31.0 \%)\end{array}$ & $\begin{array}{c}\text { Medium } \\
n=75(28.7 \%)\end{array}$ & $\begin{array}{c}\text { Large } \\
n=105(40.2 \%)\end{array}$ & $\begin{array}{c}\text { Total } \\
N=\mathbf{2 6 1}\end{array}$ \\
\hline \multicolumn{5}{|l|}{ Education level } \\
\hline Less than high school & $23(28.4)$ & $12(16.0)$ & $10(9.5)$ & $45(17.2)$ \\
\hline High school diploma/GED & $33(40.7)$ & $39(52.0)$ & $42(40.0)$ & $114(43.7)$ \\
\hline Trade/vocational school & $4(4.9)$ & $4(5.3)$ & $13(12.4)$ & $21(8.0)$ \\
\hline Some college/associate degree & $17(21.0)$ & $18(24.0)$ & $31(29.5)$ & $66(25.3)$ \\
\hline Bachelor's/graduate degree & $4(4.9)$ & $2(2.7)$ & $9(8.6)$ & $15(5.7)$ \\
\hline \multicolumn{5}{|l|}{ Care-related training } \\
\hline CNA & $35(43.2)$ & $39(52)$ & $74(70.5)$ & $148(56.7)$ \\
\hline LPN & 0 & $5(6.7)$ & $6(5.7)$ & $11(10.5)$ \\
\hline Medication certification & 0 & (2.7) & $10(9.5)$ & $12(11.4)$ \\
\hline \multicolumn{5}{|l|}{ Years in LTC } \\
\hline$\leq$ year & $11(13.6)$ & $7(9.3)$ & $7(6.7)$ & $25(9.6)$ \\
\hline$>1$ and $\leq 5$ years & $28(34.6)$ & $32(42.7)$ & $38(36.5)$ & $98(37.7)$ \\
\hline$>5$ and $\leq 10$ years & $20(24.7)$ & $17(22.7)$ & $29(27.9)$ & $66(25.4)$ \\
\hline$>10$ years & $22(27.2)$ & $19(25.3)$ & $30(28.8)$ & $71(27.3)$ \\
\hline \multicolumn{5}{|l|}{ Years in the facility } \\
\hline$\leq 1$ year & $34(42.0)$ & $15(20.0)$ & $29(27.6)$ & 78 (29.9) \\
\hline$>1$ and $₫$ years & $20(24.7)$ & $27(36.0)$ & $45(42.9)$ & $121(35.3)$ \\
\hline$>3$ and 5 years & $14(17.3)$ & $13(17.3)$ & $16(15.3)$ & 43 (16.4) \\
\hline$>5$ years & $13(16.1)$ & $20(26.7)$ & $15(14.3)$ & 48 (18.4) \\
\hline
\end{tabular}

J Appl Gerontol. Author manuscript; available in PMC 2019 December 04. 